http://dx.doi.org/10.35630/2199-885X/2020/10/2.Ed002

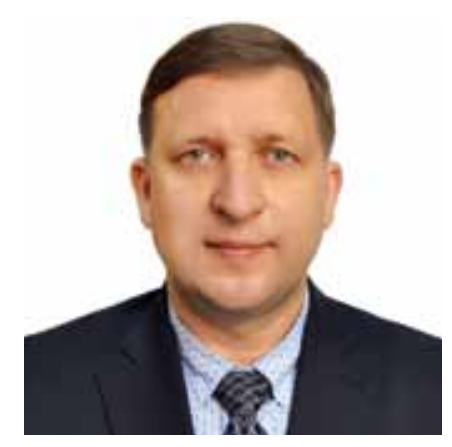

\title{
Dear clinicians, research fellows, colleagues and friends!
}

Multitude of nosological forms, their individuality and variability among different patients - all this make the process of diagnosis fairly complicated. Moreover, the outcome of a treatment depends on knowledge and skills of a doctor, which may often be insufficient.

Therefore, a special attention in clinical medicine is paid to the latest achievements in biology, physics, chemistry, as well as in engineering and electronics. Thus, objective testing methods, which are incorporated in clinical practice, extend the volume and enhance the informativeness of acquired data.

The feasibility for development of clinical laboratory diagnosis or laboratory medicine is determined by emerging technologies for in vitro testing of human biomaterials using hematologic, general clinical, parasitic, biochemical, immunological, serological and many other methods of laboratory examinations.

In modern health care system, $70 \%$ of medical decisions, practically in all clinical disciplines, are based on laboratory results. Laboratory tests are responsible for not less than $93 \%$ of objective diagnostic examinations; they are included into the programs of obligatory checkups as well as in standards of medical treatments for most somatic symptoms.

The results of laboratory tests have a special priority for early and accurate detection of the signs of pathological conditions. They establish an evidencebased approach to solution of social and legal issues. The contribution of laboratory tests to the development of evidence medicine is indisputable.

Among most important components of laboratory medicine, a special place belongs to the pathobiological section, which is based on fundamental theoretical knowledge and advancements, a section of laboratory (clinical) analytics dealing with systemization of the means and methods for conducting the tests. It also contains a section of clinical-laboratory diagnosis, which gives evidence on the informativeness of laboratory data and solutions for concrete medical problems.

Interdisciplinary interaction between laboratory clinical diagnosis and general therapy, gastroenterology, pulmonology, endocrinology, pediatrics, cardiology, neurology, nephrology, dentistry takes place as a rapidly growing cooperation, promoting incorporation of innovative technologies, extension of medical knowledge and update of methods of early diagnosis.

Today, laboratory diagnostics demands even more differentiation of the object and methods of diagnostics. Therefore, it is so important to join the efforts of laboratory doctors and clinicians brought together by an interdisciplinary approach.

Adding the section Clinical Laboratory Diagnostics to the journal Archiv Euromedica will highlight new publications on such subjects as preclinical verification of pathology, differential diagnosis of clinical manifestations, assessing the efficiency of preventive and therapeutic measures. 